\title{
Fluorescent Advanced Glycation End Products in the Detection of Factual Stages of Cartilage Degeneration
}

\author{
M. HANDL ${ }^{1,3}$, E. FILOVÁ ${ }^{2,3}$, M. KUBALA ${ }^{4}$, Z. LÁNSKÝ ${ }^{5}$, L. KOLÁČNÁ ${ }^{2,3}$, \\ J. VORLÍČEK ${ }^{6}$, T. TRČ ${ }^{1}$, M. PACH ${ }^{7}$, E. AMLER ${ }^{2,3}$ \\ ${ }^{1}$ Orthopedic Clinic, Second Faculty of Medicine, Charles University in Prague, Czech Republic, ${ }^{2}$ Institute of \\ Biophysics, Second Faculty of Medicine, Charles University in Prague, Czech Republic, ${ }^{3}$ Department of \\ Tissue Engineering, Institute of Experimental Medicine, Academy of Sciences of the Czech Republic, Prague, \\ Czech Republic, ${ }^{4}$ Department of Biophysics, Faculty of Science, Palacky University in Olomouc, Czech \\ Republic, ${ }^{5}$ Department of Protein Structure, Institute of Physiology, Academy of Sciences of the Czech \\ Republic, Czech Republic, ${ }^{6}$ Department of Biomathematics, Institute of Physiology, Academy of Sciences of \\ the Czech Republic, Prague, Czech Republic and ${ }^{7}$ Department of Orthopaedics, Faculty of Medicine and \\ Dentistry, Palacky University in Olomouc, Czech Republic
}

Received January 2, 2006

Accepted February 27, 2006

On-line available March 23, 2006

\begin{abstract}
Summary
Patients treated for knee disorders were included in this study. They were examined clinically (Lequesne and Tegner scores) and by standard X-ray investigation. Patients underwent a surgical procedure, either arthroscopy or knee replacement. At the initial phase of surgery, a sample of cartilage was taken for laboratory examination. Progression of the disorder and the clinical examination was correlated with the actual state of the cartilage using a novel fluorescence approach. The intrinsic fluorescence of cartilages was shown as a suitable and sensitive method for detection of the actual state of cartilages because the correlation with X-ray examination and clinical status was found. Intrinsic fluorescence properties of cartilages from patients with chondropathy and osteoarthritis were described and found to be age-dependent. We also observed a higher concentration of advanced glycation end products due to inflammatory and/or degenerative processes in the cartilage. In addition, acute pathological changes due to diseases such as meniscal lesions or anterior cruciate ligament rupture caused a significant increase of formation of advanced glycation end products even in the group of young patients. In fact, such an observation could be crucial and important for the detection of knee conditions suspected of early meniscal and/or ACL lesions especially among young patients.
\end{abstract}

\section{Key words}

Nonenzymic glycation $\bullet$ Fluorescence detection $\bullet$ Cartilage $\bullet$ Knee joint

\section{Introduction}

Knee disorders and osteoarthritis of joints belong to the most frequent diseases. Cartilage breakdown due to these diseases results in severe pain and disability. In addition to chondrocytes, cartilage 
comprises a highly hydrated proteoglycan matrix with a collagen network. Cartilage collagen fibers exhibit a very high tensile strength that increases with age (Parry et al. 1978, Jones 1991). Biomechanical properties of cartilage are closely related to collagen fiber distribution and orientation (Wu and Herzog 2002). The age-dependent changes of mechanical properties involve two distinct mechanisms of cross-linking: enzymatic cross-linking and non-enzymatic glycation. Enzymatic cross-linking is controlled by lysyl oxidase, a copper-dependent enzyme. On the other hand, nonenzymatic cross-linking, referred to also as glycation, results in the appearance of advanced glycation end products (AGEs). It also increases collagen stiffness and insolubility. Production of AGEs involves the reaction of lysine with glucose and then either oxidation of the complex that leads to formation of intermolecular cross-links, such as pentosidine, or conversion of a Schiff base (Amadori product) to carboxymethyllysine (CML). Glyoxal and glucosone, products of oxidative degradation, can react with lysine and form AGEs (Dyer et al. 1991, Grandhee and Monnier 1991, Wells-Knecht et al. 1995, Bailey et al. 1998).

It has been reported that the production of AGEs can be modified in patients with chronic diseases, such as diabetes (Dyer et al. 1993, Schleicher et al. 1997, Santana et al. 2003), atherosclerosis (Hoshino et al. 1995), rheumatoid arthritis (Takahashi et al. 1997, RodriguezGarcia et al. 1998) and osteoarthritis (Chen et al. 1999). Age-dependent concentration of pentosidine, one of AGEs, has also been reported in cartilages (Sell and Monnier 1989). Immunocytochemical staining for carboxymethyllysine shows increased staining with age in cartilage (Schleicher et al. 1997). Verzijl et al. (2001) reported that the pentosidine level increased in cartilage (aggrecan) but the rate of pentosidine accumulation is three times lower than in collagen (5-fold and 15-fold increases, respectively). The higher accumulation of pentosidine in collagen may result from a higher turnover of aggrecan compared with collagen.

The aim of our study was to understand better the relationship between diseases and fluorescence properties of intrinsic fluorophores from cartilages of human patients. Both acute diseases, such as anterior cruciate ligament rupture, chondropathy or meniscectomy, and chronic processes, such as synovitis and osteoarthritis were investigated. The clinical evaluation scores were compared and correlated.

\section{Methods}

\section{Selected diagnosis, surgery, sample collection}

Patients in the study were treated according to the principles of the study design of the IGA Grant No. 8122. They underwent a surgical procedure either arthroscopically or total knee replacement. The diagnoses for the surgery were osteoathritis, chondropathy, chronic synovitis with joint effusion and injuries (cruciate ligaments, meniscal injury, chronic instability after knee spraining). The samples for further experimental investigation were prepared from cartilage remnants after the surgical treatment designated for trash.

\section{Clinical examination}

All patients underwent clinical examination when Lequesne and Tegner scores were evaluated (Tegner and Lysholm 1985, Lequesne et al. 1987). The Outerbridge scale was used during the surgical procedure for detecting the severity of chondropathy (Outerbridge 1961).

\section{$X$-ray examination}

Routine radiographic examination (standard and lateral projections in the supine position) was done in all cases before the surgery, X-ray evaluation was performed according to the Jäger and Wirth score (Jäger and Wirth 1986) using a 4-grade scale (I - initial stage, prolonged and sharpened eminentia intercondylica, II - mild stage, decrease of joint space, subchondral sclerosis, light deformity of femoral condyles, III - expressed stage, severe osteophytes, sclerocystic degeneration of subchondral bone, malalignment of joint axis, narrowing of joint space, IV - severe stage, disappearing joint space, severe osteophytes, subluxation of femur against tibia, general osteosclerotic damage of the joint).

\section{Surgical procedures and sample collection}

Both surgical procedures (arthroscopy and total knee replacement) were conducted under general or spinal anesthesia in an operating theater. After the cartilage evaluation during the initial phase of the surgery, samples of cartilage were harvested from the non-weight-bearing zone of trochlea femoris. The sharp surgical double-spoon (conchotom) was used, so that the full thickness cartilage to the subchondral lamina was obtained in rice seed size $(2 \times 2 \times 2 \mathrm{~mm}$, about 100-200 $\mathrm{mg}$ ). The procedure was performed under sterile conditions and the harvested sample from the joint was 
inserted into a syringe filled with physiological saline and transported to the laboratory in $10 \mathrm{ml}$ of physiological solution, stored at room temperature for a maximum of 12 hours. Consequently the complete treatment procedure was followed. In the post-operation period wound healing was without any complication in all cases.

\section{Cartilage cleavage and sample preparation}

The excised cartilage was incubated in $1 \%$ (w/w) acetic acid overnight and lyophilized. Then, $0.5 \mathrm{~g}$ of the cartilage was cleaved by bacterial collagenase (collagenase:collagen $=1: 100, \mathrm{~W} / \mathrm{W}$ ) in $50 \mathrm{ml}$ of the incubation solution of $160 \mathrm{mM}\left(\mathrm{NH}_{4}\right)_{2} \mathrm{CO}_{3}$ and $1.3 \mathrm{mM}$ $\mathrm{CaCl}_{2}$ (pH 7.8) for $48 \mathrm{~h}$. Afterwards the sample was used for fluorescence measurements at $37{ }^{\circ} \mathrm{C}$ in $20 \mathrm{mM}$ Tris$\mathrm{HCl}(\mathrm{pH} 7.4)$.

\section{Fluorescence measurement}

Steady-state excitation at $\lambda_{\mathrm{ex}}=335 \mathrm{~nm}$ or $\lambda_{\mathrm{ex}}=$ $370 \mathrm{~nm}$ and emission at $\lambda_{\mathrm{em}}=400 \mathrm{~nm}$ or $\lambda_{\mathrm{em}}=440 \mathrm{~nm}$ spectra were recorded on the Perkin-Elmer LS50B spectrofluorimeter. For determination of the dependence of AGEs formation on age, $600 \mu \mathrm{l}$ of the incubation solution were diluted in $2 \mathrm{ml}$ of $20 \mathrm{mM}$ Tris- $\mathrm{HCl}(\mathrm{pH}$ 7.4) and the fluorescence intensities of the solution at excitation/emission wavelengths of $335 / 400 \mathrm{~nm}$ and $370 / 440 \mathrm{~nm}$ were recorded at $37{ }^{\circ} \mathrm{C}$. Both excitation and emission bandpasses were set to $5 \mathrm{~nm}$, the signal was integrated for $5 \mathrm{~s}$. The measured fluorescence intensity values were corrected for light scattering and normalized. To normalize intrinsic fluorescence intensity of the cartilages, the total protein concentration was determined for each sample and the relative fluorescence intensity to the protein concentration was estimated. All measurements were done and recorded at $37^{\circ} \mathrm{C}$.

Steady-state fluorescence intensity was corrected for protein concentration similarly as described by Amler et al. (1996).

\section{Statistical analysis}

Nonparametric regression analysis of variance for correlation and nonparametric Spearman coefficient for statistical significance were determined for each diagnosis. Statistical significance of the difference between more than two groups was determined by nonparametric Kruskal-Wallis ANOVA and between two groups by nonparametric Mann-Whitney U Test. Biased samples were omitted. $\mathrm{P}<0.05$ values were considered significant. Groups containing 2 patients were evaluated only by nonparametric regression analysis of variance.

\section{Results}

\section{Group of patients, diagnosis and sample harvesting}

A total of 125 patients (68 men and 57 women) were involved in our approved study. They were treated either for an acute injury of the knee or for chronic chondropathy or osteoarthritis. The age of patients ranged from 15 to 85 years (the mean age was 47.6 years).

Five main diagnoses, such as degenerative osteoarthritis, meniscal lesions, anterior cruciate ligament lesion, chondropathy and synovitis were followed (Table 1). Patients with chondropathy were taken as a control group to patients of similar age treated for other diagnoses, e.g. acute injury or osteoarthritis (OA).

Table 1. Groups of patients according to age and diagnosis

\begin{tabular}{llllll}
\hline Age & ACL & CH & ME & S & OA \\
\hline $15-25$ & 6 & 7 & 2 & & \\
$26-40$ & 19 & 8 & 11 & 1 & 1 \\
$41-55$ & & 4 & 12 & 5 & 5 \\
$56-70$ & & & & 1 & 18 \\
$71-85$ & & & & & 25 \\
\hline
\end{tabular}

$\mathrm{OA}$, degenerative osteoarthritis; $\mathrm{ME}$, meniscal lesions; $\mathrm{ACL}$, anterior cruciate ligament; $\mathrm{CH}$, chondropathy; $\mathrm{S}$, synovitis

Two sets of intrinsic fluorophores characterized the cartilage samples. The samples were digested and prepared for fluorescence studies as described in the Methods. Steady-state fluorescence spectra were measured. In fact, we detected at least two sets of intrinsic fluorophores in each sample, probably as a consequence of non-enzymatic glycation products. A strong fluorescence intensity of the sample was observed at the excitation wavelength of $\lambda_{\mathrm{ex}}=335 \mathrm{~nm}$ and the emission wavelength of $\lambda_{\mathrm{em}}=400 \mathrm{~nm}$ (referred to as fluorescence $335 / 400$ in this paper). Another fluorescence peak (referred to below as fluorescence 370/440) was observed at excitation wavelength of $\lambda_{\text {ex }}=370 \mathrm{~nm}$ and emission wavelength of $\lambda_{\mathrm{em}}=440 \mathrm{~nm}$. The measured fluorescence intensity values were corrected for light scattering and normalized. To normalize intrinsic fluorescence intensity of the cartilages, the total protein concentration was determined for each sample and the relative fluorescence intensity to the protein 
concentration was estimated. All measurement were performed one and recorded at $37{ }^{\circ} \mathrm{C}$.

Age-related increase of intrinsic fluorescence intensity from cartilages of chondropathy patients

Intrinsic fluorescence intensity of cartilage samples increased with age. This increase of fluorescence was observed for both sets of fluorophores (for 335/400 fluorescence as well as for 370/440 fluorescence) and the average value confirmed for each measurement was determined from triplicates.

A significant (eleven-fold) increase of 335/400 fluorescence with age in the group with chondropathy (Fig. 1) was found. In fact, the obtained data could be described as a linear increase of the 335/400 fluorescence intensity with age (with the correlation coefficient $r=0.943$ ). Similar behavior was observed for 370/440 fluorescence despite a lower correlation coefficient $(r=0.86)$. However, different behavior could be observed in patients with meniscal lesions, anterior cruciate ligament lesion and synovitis. Our results show

that both fluorescence intensities were significantly correlated with neither the objective findings nor the clinical tests (data not shown).

On the other hand, the 370/440 fluorescence intensity is significantly correlated with both objective findings and clinical tests (Table 2).

Intrinsic fluorescence intensity in the cartilage from patients with osteoarthritis is significantly elevated but still retains the age-related dependence

Both fluorescence intensities (335/400 fluorescence as well as $370 / 440$ fluorescence) were significantly elevated in the group of OA patients compared with the group of $\mathrm{CH}$ patients (Figs $2 \mathrm{~A}$ and 2B). Beside this important observation, fluorescence intensities of $370 / 440$ fluorescence, in the group of OA

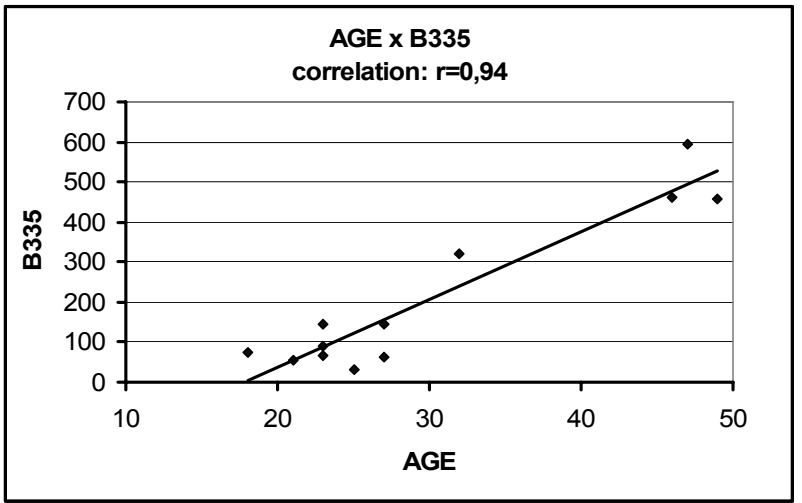

Fig. 1. Age-dependent $335 / 400$ fluorescence of pentosidine. A significant (eleven-fold) increase with age was found in the group with $\mathrm{CH}(y=16.945 \times$ age $-301.4, r=0.943, p=0.01276)$.

patients retained a significant correlation with age. In fact, a better correlation was found for the 370/440 fluorescence intensity (Fig. 3). The obtained data could be described as a linear increase as the best fitting of the measured data of the 370/440 fluorescence intensity with age even if the correlation coefficient was relatively low $(r=0.606)$.

Correlation between intrinsic fluorescence of cartilage and clinical examination

The intrinsic fluorescence intensities for both fluorescence maxima, i.e. 335/400 fluorescence as well as $370 / 440$ fluorescence, were measured in triplicate. Correlation coefficients of significance between intrinsic cartilage fluorescence and clinical X-ray finding and three examinations are summarized in Table 2. A correlation between the 335/400 fluorescence and the Xray pattern as well as between 370/440 fluorescence and the clinical examination were found. The $p$ values (presented in parentheses in Table 2) clearly suggest, a worse correlation of the 335/400 fluorescence with clinical examinations. The reason for this remains

Table 2. Correlation between $\mathrm{X}$-ray diagnosis, clinical examination and intrinsic cartilage fluorescence.

\begin{tabular}{lllllll}
\hline & X-ray & Outerbridge & $\begin{array}{l}\text { Tegner and } \\
\text { Lysholm }\end{array}$ & Lequesne & $\begin{array}{l}\mathbf{3 3 5} / \mathbf{4 0 0} \\
\text { fluorescence }\end{array}$ & $\begin{array}{l}\mathbf{3 7 0 / 4 4 0} \\
\text { fluorescence }\end{array}$ \\
\hline X-ray & 1 & $0.877(<0.0001)$ & $-0.826(<0.0001)$ & $0.626(<0.0001)$ & $0.270(0.0029)$ & $0.480(<0.0001)$ \\
Outerbridge & $0.877(<0.0001)$ & 1 & $-0.743(<0.0001)$ & $0.613(<0.0001)$ & $0.245(0.0072)$ & $0.502(<0.0001)$ \\
Tegner & $-0.826(<0.0001)$ & $-0.743(<0.0001)$ & 1 & $-0.677(<0.0001)$ & $-0.245(0.0073)$ & $-0.527(<0.0001)$ \\
Lequense & $0.626(<0.0001)$ & $0.613(<0.0001)$ & $-0.677(<0.0001)$ & 1 & $0.089(0.3394)$ & $0.38(<0.0001)$ \\
\hline
\end{tabular}

Correlation coefficients and appropriate $p$ values (in parentheses) for $\mathrm{X}$-ray findings and clinical examinations and intrinsic cartilage fluorescence. $370 / 440$ fluorescence examination appeared to be significant, correlation for both methods, i.e. $335 / 400$ fluorescence and $370 / 440$ fluorescence, is evident. 
not clear and needs further examination. Though the correlation of the $335 / 400$ fluorescence intensities with clinical examinations appeared to be significant, a correlation for 370/440 fluorescence is even more evident. The $p$ values in parentheses show that corrected $370 / 440$ fluorescence values can be well correlated with $\mathrm{X}$-ray findings as well as with all three clinical examinations (Table 2).

$\mathbf{A}$

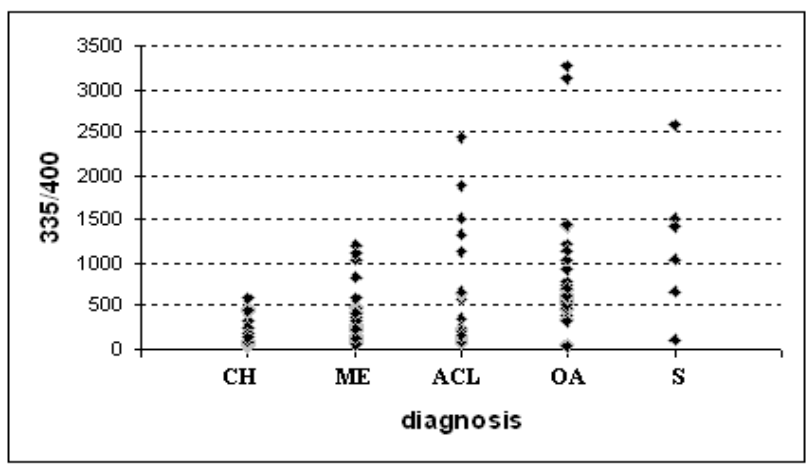

B

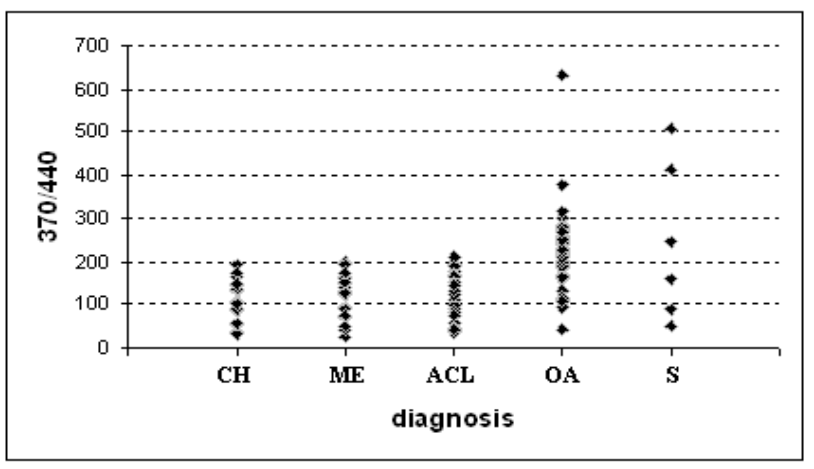

Fig. 2. Intrinsic fluorescence intensities from cartilages of patients with different diagnosis. A. 335/400 fluorescence from cartilages of patients with $\mathrm{CH}$ (20 patients; $\mathrm{I}_{\mathrm{f}}=213 \pm 176$ a.u.); ME (25 patients; $I_{f}=400 \pm 316$ a.u.); ACL (24 patients; $I_{f}=537$ \pm 610 a.u.); OA (49 patients; $I_{\mathrm{f}}=724 \pm 224$ a.u.); S (7 patients; $I_{f}=1225 \pm 849$ a.u.). B. $370 / 440$ fluorescence from cartilages of patients with $\mathrm{CH}$ (20 patients; $\mathrm{I}_{\mathrm{f}}=102 \pm 53$ a.u.); $\mathrm{ME}$ (25 patients; $I_{f}=137 \pm 68$ a.u.); $A C L$ (24 patients; $I_{f}=136 \pm 76$ a.u.); OA (49 patients; $I_{\mathrm{f}}=224 \pm 89$ a.u.); S (7 patients; $\mathrm{I}_{\mathrm{f}}=$ $244 \pm 183$ a.u.). According to the t-test, patients with $O A$ significantly differ $(p<0.0001)$ from those with $\mathrm{CH}$.

Significantly increased intrinsic fluorescence intensity was observed in the cartilage of patients with osteoarthritis, meniscal lesions, anterior cruciate ligament lesion and synovitis.

A correlation of the fluorescence intensity of samples from patients with chondropathy (335/400 fluorescence) and osteoarthritis (370/440 fluorescence) with age was observed and described in this study. The groups of patients with osteoarthritis, meniscal lesions, anterior cruciate ligament lesion and synovitis were not affected by age. However, a significantly higher 335/400 and 370/440 fluorescence intensities were found in the group of patients with anterior cruciate ligament lesion compared to the group of $\mathrm{CH}$ patients (Figs. 2A and 2B). Such an increase was observed even in the group of youngest patients (15-25 years old).

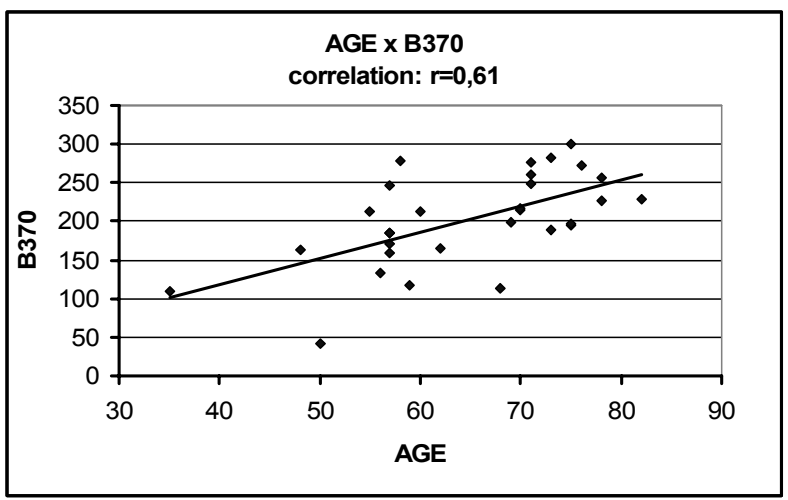

Fig. 3. Age-dependent $370 / 440$ fluorescence of nonspecific glycation product. Patients with $\mathrm{OA}$ showed a significant correlation of nonspecific glycation product fluorescence intensity with age $(y=3.3742 \times$ age $-16.67, r=0.606, p=0.00018)$.

\section{Discussion}

Frequent diseases, such as chondropathy or injuries of the knee joint, can affect the intraarticular environment and result in articular degeneration. Those sequels can be verified according to various classification schemes. To the most commonly accepted systems describing the severity of hyaline cartilage damage belong those from Outerbridge (1961) and Noyes and Stabler (1989). There are, however, certain limitations and deficiencies in both systems. Outerbridge divided the changes into four grades. Noyes and Stabler use simple terms for describing articular cartilage abnormalities - the description of the articular surface, the extent (depth) of involvement, the diameter of the lesion, and its location. According to this system, in spite of being somewhat qualitative and subjective, one can evaluate the observed articular cartilage changes.

Clinical examination indicates the functional perspective of a joint. There are many schemes and evaluation systems that can mutually differ. The algofunctional index (Lequesne et al. 1987) and the score (Tegner and Lysholm 1985) belong to the most commonly used schemes for the evaluation of the the functional joint status. The prevalence of osteoarthritis is 
supposed to increase with age.

A correlation and substitutability among the clinical tests and X-ray findings was observed during this study. Based on our results we concluded that a significant mutual correlation exists between the individual clinical tests of Outerbridge (1961) and of Tegner and Lysholm (1985). These clinical tests are supposed to be mutually substitutable at least for the diagnosis of degenerative osteoarthritis, meniscal lesions, rupture of anterior cruciate ligament, chondropathy and synovitis (Table 2).

In addition, the results of X-ray examination significantly correlated with the clinical tests. There was a highly significant correlation among the clinical tests and X-ray examinations. Such a correlation indicates that the clinical tests are well chosen and also that the subjective complaints and ailments of the majority of patients reflected the objective diagnosis. Table 2 also suggests a somewhat worse correlation with the clinical examination according to Tegner and Lysholm (1985). The reason why this test differed from the other two could be of potential interest in the future.

Our findings indicate that both the 335/400 and $370 / 440$ fluorescence intensities are suitable markers for estimation of cartilage deterioration and can characterize patients with osteoarthritis, meniscal lesions, anterior cruciate ligament lesion or synovitis.

We found a mutual correlation among the clinical tests and X-ray findings. However, the highest interest was to observe a correlation of clinical tests with the cartilage intrinsic fluorescence intensity. Such information would be valuable for the real biological status determination of cartilage without reference to age.

In digested articular cartilages, we found two groups of intrinsic fluorophores with fluorescence maxima at $\lambda_{\mathrm{em}}=400 \mathrm{~nm}$ and $\lambda_{\mathrm{em}}=440 \mathrm{~nm}$ (excitation at $\lambda_{\mathrm{ex}}=335 \mathrm{~nm}$ and $\lambda_{\mathrm{ex}}=370 \mathrm{~nm}$, respectively). The ratio of the $335 / 400 \mathrm{~nm}$ fluorophore concentration with respect to protein increased linearly with age. This is demonstrated in the group of patients with chondropathy which is, in fact, reported to be an initial ailment of cartilage. This group substituted in our study the control group of healthy patients for a simple reason: the group of healthy patients could be insignificant from a statistical point of view. The relative 335/400 fluorescence intensity was age-related.

According to hitherto published reports, the production of AGEs can be modified in patients with chronic diseases, such as diabetes, atherosclerosis, and osteoarthritis in many tissues (Dyer et al. 1993, Chen et al. 1999). Accumulation of AGEs especially in articular cartilage has been described during development of osteoarthritis (DeGroot et al. 2001). Non-enzymatic cross-linking has been reported to increase collagen stiffness and insolubility due to formation of non-enzymatic glycation products (Bailey et al. 1998), which were also responsible for the appearance of intrinsic fluorophores. Increasing the amount of intrinsic fluorophores with age caused, naturally, higher fluorescence intensity observed in our study. Consequently, it can be concluded that the accumulation of AGEs increased with age. This is in agreement with the earlier observation of increased pentosidine levels in cartilage aggrecan (Uchiyama et al. 1991, Verzijl et al. 2001).

The linear age-dependent increase of 335/400 fluorescence is in good agreement with the observation in healthy tissues (Sell and Monnier 1989, Uchiyama et al. 1991). In contrast, a significantly lower amount of non-enzymatic glycation products was formed in aggrecan of OA patients (Verzijl et al. 2001). It can be supposed that the age-related increase in cartilage AGEs levels may be responsible, at least in part, for the agerelated decline in the synthetic capacity of cartilage and concentration of the nonspecific glycation products increases in $\mathrm{OA}$ patients with age. Interestingly, no differences were found in patients with other diagnoses (irrespective of their age). It should be mentioned that OA patients exhibited a significantly higher fluorescence intensity levels compared with the group of chondropathy patients. The generally higher fluorescence which further increased with age probably reflected a higher amount of nonspecific glycation products caused by degenerative changes in OA cartilage. In addition, it may also be due to ageing which can independently increase the amount of AGEs.

The results also support the view that an increased level of degradation products in ACL is similar to the level of OA changes at a higher age. ACL in young patients increased the formation of non-enzymatic glycation products in comparison with the chondropathy group. Moreover, no correlation of non-enzymatic glycation products with age in other diagnoses had indicated that the acute processes may stimulate a higher non-enzymatic glycation product formation in young patients. Thus, it can be concluded that hyaline cartilage in the knee joint after repeated inflammations or injuries contains a concentration of AGEs which is comparable to 
the concentration in healthy older patients. In fact, such an observation could be of crucial importance for the detection of knees suspected of early meniscal and/or ACL lesions especially among young patients.

\section{Acknowledgements}

This work was supported by the Internal Grant Agency of the Ministry of Health of the Czech Republic grant (IGA) No. 8122-3/2004, 210060, the Grant Agency of AS CR grants No. 1ET400110403 and IAA500390702, the Grant Agency of the Charles University in Prague grants No. 46/2005, 121/2005, the Ministry of Education, Youth and Sport of the Czech Republic (NPV II 2B06130) and the Cardiovascular Research Center (1M6798582302).

\section{References}

AMLER E, ABBOTT A, MALAK H, LAKOWICZ J, BALL WJ, Jr.: The carbohydrate moieties of the beta-subunit of $\mathrm{Na}^{+}, \mathrm{K}^{+}$-ATPase: their lateral motions and proximity to the cardiac glycoside site. Biophys J 70: 182-193, 1996.

BAILEY AJ, PAUL RG, KNOTT L: Mechanisms of maturation and ageing of collagen. Mech Ageing Dev 106: 1-56, 1998.

CHEN JR, TAKAHASHI M, SUZUKI M, KUSHIDA K, MIYAMOTO S, INOUE T: Comparison of the concentrations of pentosidine in the synovial fluid, serum and urine of patients with rheumatoid arthritis and osteoarthritis. Rheumatology (Oxford) 38: 1275-1278, 1999.

DEGROOT J, VERZIJL N, JACOBS KM, BUDDE M, BANK RA, BIJLSMA JW, TEKOPPELE JM, LAFEBER FP: Accumulation of advanced glycation endproducts reduces chondrocyte-mediated extracellular matrix turnover in human articular cartilage. Osteoarthritis Cartilage 9: 720-726, 2001.

DYER DG, BLACKLEDGE JA, THORPE SR, BAYNES JW: Formation of pentosidine during nonenzymatic browning of proteins by glucose. Identification of glucose and other carbohydrates as possible precursors of pentosidine in vivo. J Biol Chem 266: 11654-11660, 1991.

DYER DG, DUNN JA, THORPE SR, BAILIE KE, LYONS TJ, MCCANCE DR, BAYNES JW: Accumulation of Maillard reaction products in skin collagen in diabetes and aging. J Clin Invest 91: 2463-2469, 1993.

GRANDHEE SK, MONNIER VM: Mechanism of formation of the Maillard protein cross-link pentosidine. Glucose, fructose, and ascorbate as pentosidine precursors. J Biol Chem 266: 11649-11653, 1991.

HOSHINO H, TAKAHASHI M, KUSHIDA K, OHISHI T, KAWANA K, INOUE T: Quantitation of the crosslinks, pyridinoline, deoxypyridinoline and pentosidine, in human aorta with dystrophic calcification. Atherosclerosis 112: 39-46, 1995.

JÄGER M, WIRTH CJ: Praxis der Orthopädie. Thieme, Stuttgart, 1986, p 980.

JONES PN: On collagen fibril diameter distributions. Connect Tissue Res 26: 11-21, 1991.

LEQUESNE MG, MERY C, SAMSON M, GERARD P: Indexes of severity for osteoarthritis of the hip and knee. Validation-value in comparison with other assessment tests. Scand J Rheumatol Suppl 65: 85-89, 1987.

NOYES FR, STABLER CL: A system for grading articular cartilage lesions at arthroscopy. Am J Sports Med 17: $505-$ $513,1989$.

OUTERBRIDGE RE: The etiology of chondromalacia patellae. J Bone Joint Surg Br 43: 752-757, 1961.

PARRY DA, CRAIG AS, BARNES GR: Tendon and ligament from the horse: an ultrastructural study of collagen fibrils and elastic fibres as a function of age. Proc R Soc Lond B Biol Sci 203: 293-303, 1978.

RODRIGUEZ-GARCIA J, REQUENA JR, RODRIGUEZ-SEGADE S: Increased concentrations of serum pentosidine in rheumatoid arthritis. Clin Chem 44: 250-255, 1998.

SANTANA RB, XU L, CHASE HB, AMAR S, GRAVES DT, TRACKMAN PC: A role for advanced glycation end products in diminished bone healing in type 1 diabetes. Diabetes 52: 1502-1510, 2003.

SELL DR, MONNIER VM: Structure elucidation of a senescence cross-link from human extracellular matrix. Implication of pentoses in the aging process. $J$ Biol Chem 264: 21597-21602, 1989.

SCHLEICHER ED, WAGNER E, NERLICH AG: Increased accumulation of the glycoxidation product N(epsilon)(carboxymethyl)lysine in human tissues in diabetes and aging. J Clin Invest 99: 457-468, 1997.

TAKAHASHI M, SUZUKI M, KUSHIDA K, MIYAMOTO S, INOUE T: Relationship between pentosidine levels in serum and urine and activity in rheumatoid arthritis. Br J Rheumatol 36: 637-642, 1997. 
TEGNER Y, LYSHOLM J: Rating systems in the evaluation of knee ligament injuries. Clin Orthop Relat Res 198: 4349, 1985.

UCHIYAMA A, OHISHI T, TAKAHASHI M, KUSHIDA K, INOUE T, FUJIE M, HORIUCHI K: Fluorophores from aging human articular cartilage. J Biochem (Tokyo) 110: 714-718, 1991.

VERZIJL N, DEGROOT J, BANK RA, BAYLISS MT, BIJLSMA JW, LAFEBER FP, MAROUDAS A, TEKOPPELE JM: Age-related accumulation of the advanced glycation endproduct pentosidine in human articular cartilage aggrecan: the use of pentosidine levels as a quantitative measure of protein turnover. Matrix Biol 20: 409-417, 2001.

WELLS-KNECHT MC, THORPE SR, BAYNES JW: Pathways of formation of glycoxidation products during glycation of collagen. Biochemistry 34: 15134-15141, 1995.

WU JZ, HERZOG W: Elastic anisotropy of articular cartilage is associated with the microstructures of collagen fibers and chondrocytes. J Biomech 35: 931-942, 2002.

\section{Corresponding author}

M. Handl, Orthopedic Clinic, University Hospital Motol, V Úvalu 84, 15018 Prague 5, Czech Republic. Fax:+420 224

432 820. E-mail: milan.handl@1fmotol.cuni.cz 